\title{
Progress towards Non-Intrusive Optical Measurement of Gas Turbine Exhaust Species Distributions
}

\author{
Paul Wright \\ David McCormick \\ Krikor Ozanyan \\ The University of Manchester \\ Oxford Road \\ Manchester, UK \\ +441613064785 \\ paul.wright@ manchester.ac.uk
}

\author{
Mark Johnson \\ John Black \\ Rolls-Royce plc \\ Test and Measurement Engineering \\ Derby, UK, \\ +441332 247138 \\ Mark.Johnson3@Rolls-Royce.com
}

\author{
Edward Fisher \\ Andrea Chighine \\ Nick Polydorides \\ Hugh McCann \\ School of Engineering \\ The King's Buildings \\ The University of Edinburgh \\ Edinburgh, UK \\ +44 1316505566 , \\ h.mccann@ed.ac.uk
}

\author{
Yutong Feng \\ Khouler Khan \\ Paul Bastock \\ Fuqiang Jia \\ Dan Hewak \\ Johan Nilsson \\ Optoelectronics Research Centre \\ University of Southampton \\ Highfield, \\ Southampton, UK \\ +442380 593101 \\ jn@orc.soton.ac.uk
}

\author{
Michael Lengden \\ David Wilson \\ Ian Armstrong \\ Thomas Benoy \\ Walter Johnstone \\ University of Strathclyde \\ 204 George Street \\ Glasgow, UK \\ +44 (0)141548 2641 \\ w.johnstone@strath.ac.uk
}

\begin{abstract}
We report on the development of three systems intended to provide fast, non-intrusive measurement of crosssectional distributions of pollutant species within gas turbine exhaust flows, during ground-based testing. This research is motivated by the need for measurement systems to support the introduction of technologies for reducing the environmental impact of civil aviation. Tomographic techniques will allow estimation of the distributions of $\mathrm{CO}_{2}$, unburnt hydrocarbons (UHC), and soot, without obstruction of the exhaust, bypass or entrained flows, from measurements made in a plane immediately aft of the engine.
\end{abstract}

We describe a $\mathrm{CO}_{2}$ imaging system that performs wavelength modulation spectroscopy (WMS) simultaneously on 126 beam paths. Its novel architecture uses a Tm-doped fiber amplifier to generate sufficient optical power for the entire beam array (>3 W) from a single $1997.2 \mathrm{~nm}$ diode-laser seed, reducing cost and enabling fully parallel detection and signal recovery. Various optical propagation issues are considered, including those arising from the varying degrees of interaction with the exhaust flow that exist within the beam array, as well as pointing errors arising from the limited rigidity of the measurement system's structure.

We also report first steps towards a similar UHC measurement system, operating in the mid-infrared (MIR) region and targeting partially decomposed or oxidized fuel constituents, including formaldehyde and propene. Progress towards the chalcogenide glasses and fibers, needed for light delivery and/or amplification at these wavelengths is described.
Finally, we report on the development status of a tomographic soot imaging system, based on laser induced incandescence (LII). We have demonstrated both long (192 ns) and short (17 ns) pulse variants of LII using fiber laser sources. Single path tests on a laboratory soot generator and, in the long pulse case, on a jet engine have confirmed that the energy and beam quality available from the fiber lasers is sufficient to enable an autoprojection approach, using just two intensified CCD cameras having 'near-orthogonal' views, with respect to the excitation laser.

\section{TABLE OF CONTENTS}

1. INTRODUCTION ......................................................2

2. SYSTEM OVERVIEW .......................................3

3. OPTICAL CONSIDERATIONS ...............................3

4. $\mathrm{CO}_{2}$ MEASUREMENT .........................................5

5. UHC MEASUREMENT ........................................7

6. SOOT MEASUREMENT..........................................8

7. SUMMARY .........................................................99

ACKNOWLEDGEMENTS....................................10

REFERENCES....................................................10

BIOGRAPHY .............................................................11 


\section{INTRODUCTION}

The past two decades have seen public concern over the environmental impact of civil aviation increase markedly, to the point where it has the potential to significantly inhibit the sector's growth. In 2010, the UK airport operator BAA was forced to drop plans for expansion at Heathrow and Stansted, in the face of strong local opposition and a consequent loss of government backing for the proposal [1]. Regulatory pressure on in-flight emissions is also on the increase; the EU Emissions Trading Scheme, applicable to flights within the EEA, commenced in 2010 and imposes a tradable diminishing cap on $\mathrm{CO}_{2}$ emissions. Technological solutions are only one aspect of achieving more sustainable aviation [2] but are able to play an important role in both addressing public concerns and meeting regulatory targets. Many alternative sustainability measures involve constrained or reduced air travel, which is unattractive from an industry perspective and divides public opinion regarding the appropriate balance between environmental and economic priorities. In contrast to this, the ability of technological solutions - whether to do with the engines, airframe or use of alternative fuels - to reduce per $\mathrm{km}$ emissions makes them almost universally popular; it is hard to argue against improved efficiency.

The introduction of new technologies against a regulatory backdrop can, however, be problematic. In some cases, established measurement methods are unable to keep pace with technological development. Within the aviation sector, smoke number [3] remains the industry basis of regulatory standards relating to smoke emissions from gas turbine engines, even though modern engines are rapidly approaching the limits of its sensitivity [4]. Other industries subject to strong environmental emissions regulation have experienced similar situations. The automotive sector has sometimes found itself poorly equipped to undertake the measurements necessary to meet evolving standards. Euro 6 (September 2014) [5] imposes particulate matter limits that are just $3.6 \%$ of those imposed at the Euro standard series' inception in 1992. The same period has seen similar reductions in permissible levels of other species and the regulation of $\mathrm{NO}_{\mathrm{X}}$ and hydrocarbon emissions. A particularly undesirable situation can occur when regulation itself moves ahead of the means to verify compliance, a situation that some would argue exists in the waste incineration industry in regard to combustion chamber gas temperature. The rationale here was that the introduction of regulation would provide the demand necessary to drive commercial instrument development. In reality, no wholly satisfactory solution has been realized and compliance is often less-than-convincingly demonstrated using the defense of 'best available technology' and considerable reliance on computational fluid dynamics modelling [6]. Returning to the aviation sector, it is clearly desirable that appropriate measurement tools are developed to support the introduction of those innovations in engine and fuel technology that must ultimately reduce the environmental impact of civil aviation.
Extractive techniques presently remain the mainstay of emissions measurement within the sector, despite their significant limitations. They offer very limited temporal resolution, restricting their application to quasi-static situations, and their high extraction to measurement latency effectively precludes measurements involving transient species. Where spatial resolution is available, for example by mechanical scanning of the sampling point, the information is typically not simultaneous across the flow field. Such arrangements can also affect other measurements, e.g. acoustics, precluding their simultaneous use. Line-of-sight optical techniques are latency free and have obvious potential for improved temporal resolution but they have enjoyed limited take up, perhaps on account of the need to interpret path integrated absorption measurements.

In this paper, we will provide an overview of a series of instruments currently being developed to enhance existing measurement capability in respect of aero engine emissions. The work is being undertaken by the FLITES consortium, which comprises four leading UK universities (Strathclyde, Southampton, Edinburgh, and Manchester) working in conjunction with industrial partners Shell, Rolls-Royce, OptoSci, Fianium and Covesion. The instruments bring together the consortium's expertise in fiber laser systems, tunable diode laser absorption spectroscopy (TDLAS), laser induced incandescence (LII) and optical tomography to address the measurement of cross-sectional distributions of $\mathrm{CO}_{2}$, soot and UHCs in jet exhaust plumes. By overcoming the path-integration limitation associated with conventional line-of-sight optical diagnostics, this combination of technologies has the potential to deliver non-intrusive emissions measurement with hitherto unavailable spatiotemporal resolution. The program also includes preliminary work on adding $\mathrm{NO}_{\mathrm{X}}$ measurement capability, in anticipation of further technological progress in the midIR region.

The systems will initially be used in a development test bed context, although their design means that on-wing groundbased tests will ultimately be practicable, suggesting potential application to in-service diagnostics. In contrast to many instrument development studies, the FLITES systems will be applied to current generation engines, with already low emissions. Following validation on conventional jet fuels, the systems will be used in tests of a number of biofuels to illustrate their utility in supporting technology adoption. As Bainbridge [7] notes, “... sustainable alternative fuels will play a central role in the future of aviation ... there is greater work involved in the assessment of data gained from these sources as opposed to those with standard emission factors."

In this paper, we first provide an overview of the manner in which the systems will be deployed, followed by consideration of the difficulties associated with cross-plume optical measurements. We will then describe the proposed design and current implementation status of each system. 
programme is being carried out by the FLITES consortium (see www.flites.eu).

\section{SYSTEM OVERVIEW}

Within the test bed, the systems are intended to be operated immediately aft of the engine, before the exhaust flow enters the detuner. In order that the systems do not affect any concurrent measurements, their components are all positioned outside of the exhaust, bypass and entrained flows, necessitating optical path lengths of at least $7 \mathrm{~m}$. The $\mathrm{CO}_{2}$ and UHC systems are based on infrared absorption tomography techniques [8,9] and will be mounted on a dodecagonal gantry, implementing six tomographic projection angles (Figure 1). ${ }^{1}$ Optical field-of-view considerations mean that some elements of the LII-based soot measurement system are mounted somewhat further from the plume axis. Although the systems are not fully coplanar, at typical flow velocities their axial displacement corresponds to a temporal offset in the millisecond regime, slightly less than the time typically required for each spectral scan.

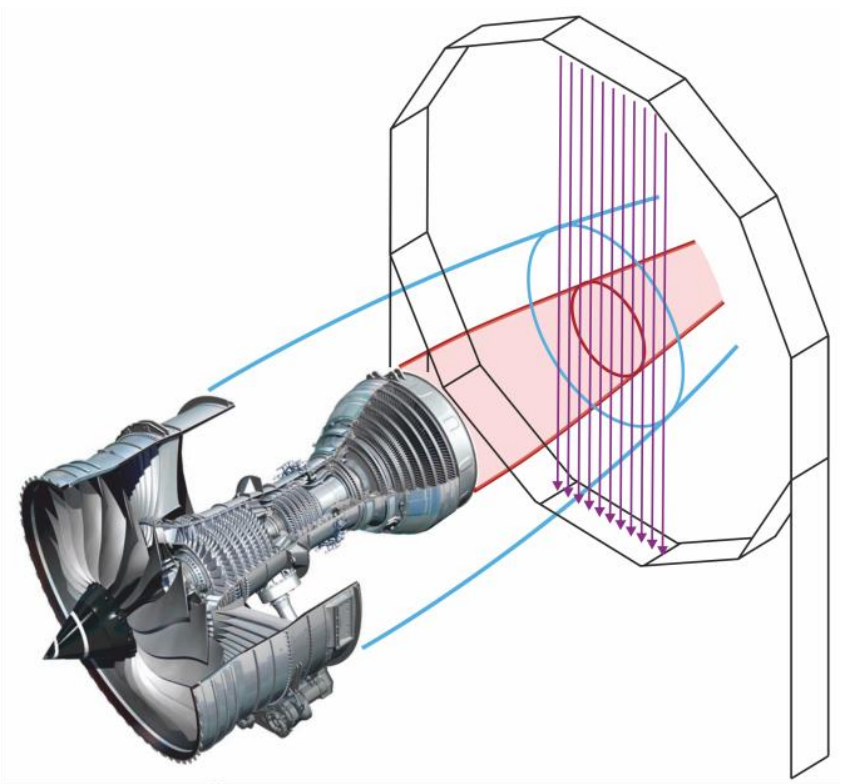

Figure 1 - Dodecagonal mounting frame for tomographic systems showing approximate extent of exhaust and bypass flows and their intersection with one projection (Trent engine cutaway (C) Rolls-Royce plc)

\section{Optical Considerations}

Optical measurements within jet exhaust flows must consider the effects of the flow itself upon beam

\footnotetext{
${ }^{1}$ This six-projection design was arrived at following detailed simulation of the measurement grid's performance, including consideration of annular burner configurations; see [10] D. McCormick, M. G. Twynstra, K. J. Daun, and H. McCann, "Optimising laser absorption tomography beam arrays for imaging chemical species in gas turbine engine exhaust plumes," in WCIPT7: 7th World Congress on Industrial Process Tomography, Krakow, Poland, 2013, pp. 505-514.
}

propagation. Indeed, one of the earliest applications of laser diagnostics was in the characterization of turbulence within jet exhaust [11]. The exhaust flow's high velocity and temperature combine to generate turbulence over a wide range of scales with strong refractive index gradients. Light propagation through turbulent atmospheres has been studied extensively in several fields, including astronomy and free space optical communications, but the resulting literature provides little quantitative insight into the expected propagation behavior in jet plumes. Of greater utility is work considering the propagation of laser-based IR countermeasures through the protected aircraft's own exhaust, e.g. $[12,13]$.

The effect of the plume on the propagating beam can be described in terms of the induced beam wander (steering) and scatter (divergence). Sirazetdinov [14] provides a detailed theoretical and experimental treatment, addressing the effects of wavelength, beam diameter and intersection angle, and has proved particularly useful. Even this has significant shortcomings for our purposes as the models developed for wander and divergence are strictly valid only for diametric intersection with the plume. As can be seen from Figure 1, a tomographic measurement grid will include beams having variable proportions of bulk and surface scales, as well as beams that remain entirely outside of the hot exhaust region. For diametric beams, the literature consensus is that plume-induced divergence is unlikely to exceed $250 \mu \mathrm{rad} \mathrm{rms}$ but will give rise to a fine grained speckle pattern and considerable scintillation. Beam wander is expected to be less significant, contributing up to $150 \mu \mathrm{rad} \mathrm{rms}$. The overall spreading effect of the plume is therefore expected to be around $300 \mu \mathrm{rad} \mathrm{rms}$, which in the proposed frame geometry would lead to $7 \mathrm{~mm}$ (pk-pk) of beam expansion. This is insufficient to pose a crosstalk problem in the proposed system geometries but does have other implications for the design of the detection system, as discussed below. Importantly, from a tomographic image reconstruction perspective, the absence of crosstalk enables the use of so-called 'hard-field' methods, in which straight line beam propagation from source to receive may be assumed.

Pointing errors, arising from the finite rigidity of the mounting structure, must also be considered. The stability of the frame has been modelled, using forcing vibrations measured in the proposed test venue, and modified to enhance its performance in this respect. Construction of the frame is due to commence in early 2015.

The combined effects of plume-induced divergence and wander, scintillation and pointing error bring surprising subtlety to the optical design of the beam path and detector. Our overall aim is optimization of the properties of the signal collected from each detector. The combination of high plume velocity and short turbulence scales largely rule out methods reliant on 'freezing' the wavefront disturbance. Even if the disturbance bandwidth were manageable, wavelength chirp is inherent in TDLAS so the effects of 
dynamic speckle are inescapable. In the absence of the plume, it would be reasonable to overfill the receiver to mitigate the effects of pointing error but this is likely to degrade signals collected in the plume's presence as increased scintillation will arise from partial sampling of the speckle pattern. Aperture averaging, in which the full beam is integrated at the detector, is highly desirable in this regard but the use of large area detectors incurs noise, bandwidth and cost penalties.

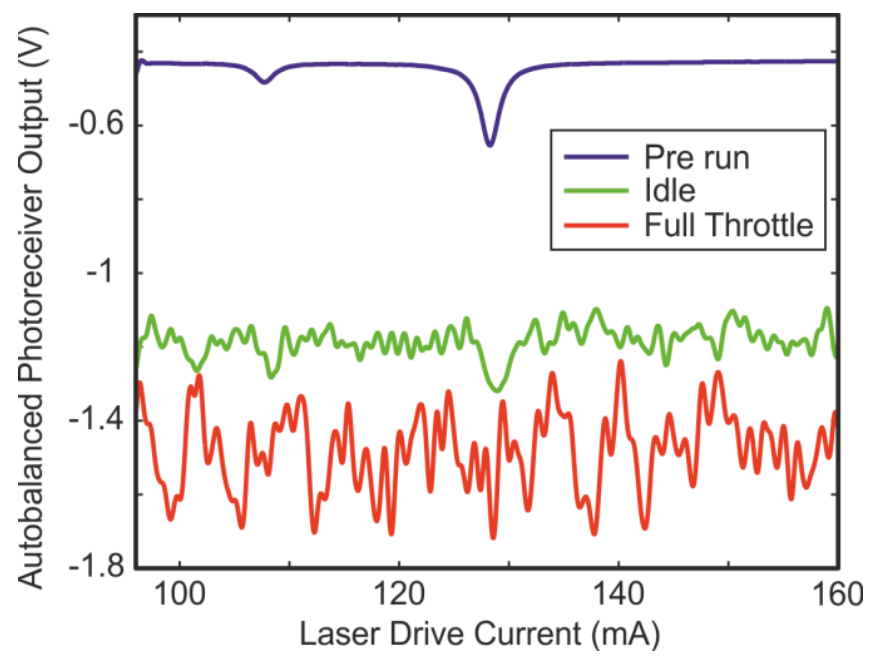

Figure 2 - Effect of jet plume on direct TDLAS measurement over a near-diametric $2.5 \mathrm{~m}$ path.

The seriousness of these issues is underlined by Figure 2, which shows three water vapor line spectra acquired using a direct TDLAS system under engine off, idle and full thrust conditions. Particularly worrying is the increase in these effects that may be seen at the required $7 \mathrm{~m}$ path length. Although the marked degradation in signal quality associated with engine operation can be mitigated by various means, for example the use of temporal averaging or wavelength modulation spectroscopy (WMS), it is evident that improving the properties of the underlying optical signals may bring about substantial overall performance gains. Our optical design goal is therefore the minimization of the detector area required to implement full aperture averaging in the face of representative levels of both pointing error and plume-induced scattering. Three basic design templates (collimated, moderately divergent, midplume focus) have been evaluated in preliminary laboratory trials, as described in [15], but the artificial thermal disturbance used is unable to replicate fully the properties of the turbulent aerojet plume. The mid-plume focus is an attempt to exploit the relatively local nature of the scattering source within the beam path. Scattering occurring close to the focal region then acts primarily to change the numerical aperture seen by the receiver, with little effect on the apparent origin of the rays. This approach does however reduce the effective beam diameter within the plume region, which will alter the balance between beam wander and scatter. As the relevant balance of pointing error and scattering is as yet unknown, the final decision will be made following initial on-frame testing, making focus adjustment an essential component of the optical design.

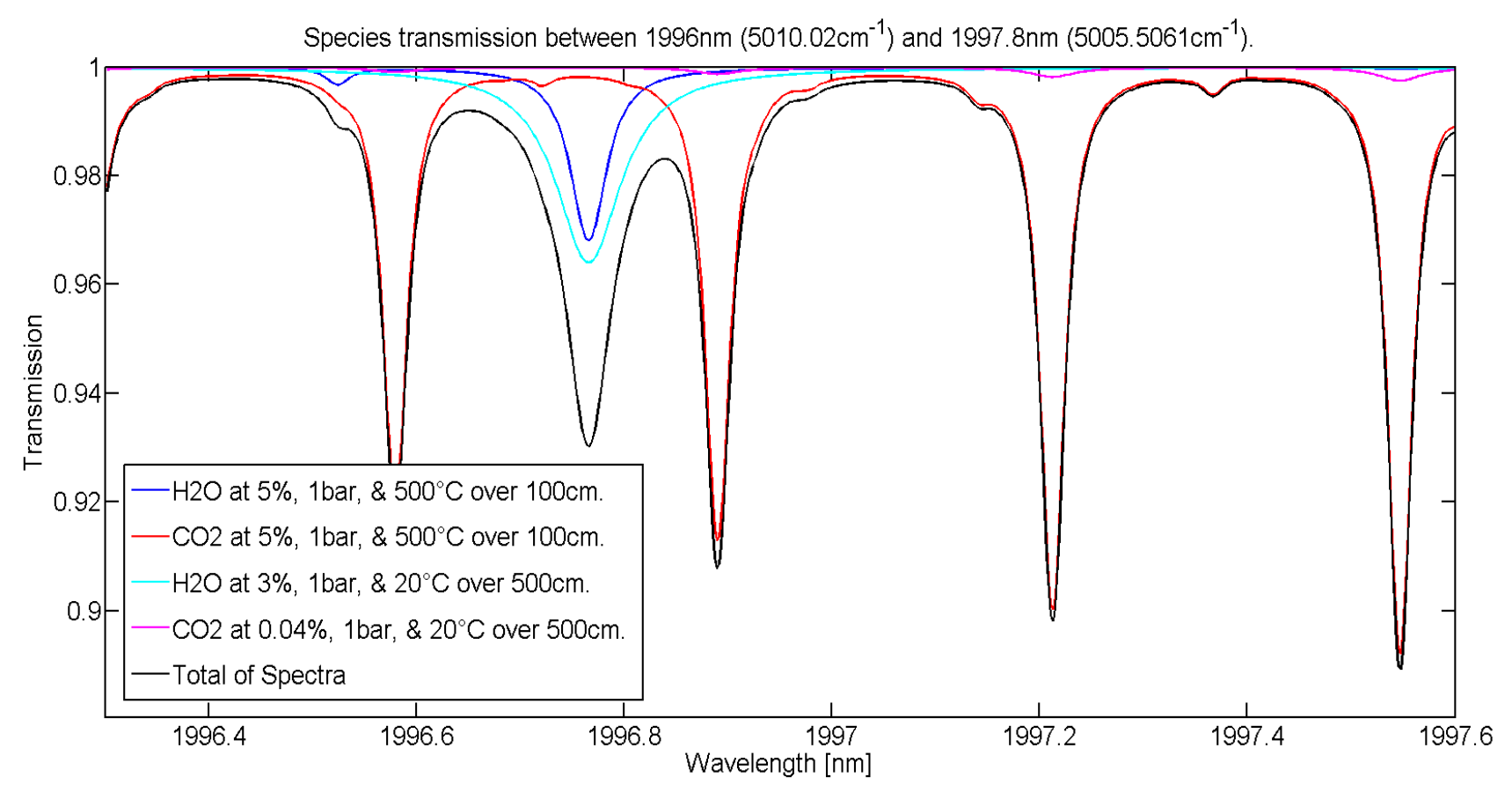

Figure $3-\mathrm{CO}_{2}$ and $\mathrm{H}_{2} \mathrm{O}$ spectra at $20^{\circ} \mathrm{C}$ and $500^{\circ} \mathrm{C}$ in the vicinity of the $\mathrm{R}(48) v 1+2 v 2+v 3$ feature 


\section{4. $\mathrm{CO}_{2}$ Measurement}

Spectroscopy

The near-IR region has abundant opportunities for monitoring major constituent species within gas mixtures, where its typically lower linestrengths can actually be an advantage. Technology for this region is mature, having benefitted from developments in the telecommunications sector, and far cheaper than that needed at mid-IR wavelengths. In the case of combustion exhaust measurement, both $\mathrm{CO}_{2}$ and $\mathrm{H}_{2} \mathrm{O}$ are accessible in the nearIR region but our focus here is on $\mathrm{CO}_{2}$ quantification, with a view to supporting implementation of the industry's strategy for carbon neutral growth from 2020 (CNG2020) [16]. The initial determination of an appropriate absorption feature was done by simulation, using the HITRAN database [17], and led to the selection of the R(48) $v 1+2 v 2+v 3$ line, located at $1997.2 \mathrm{~nm}$. This absorption feature is well isolated from cross-interference due to other species that absorb in the same region, considering both ambient and elevated temperatures (Figure 3). Other considerations were the availability of suitable lasers and the relative strength of the absorption bands. Of the three bands of $\mathrm{CO}_{2}$ in the $2 \mu \mathrm{m}$ region, $4 v 2+v 3,2 v 1+v 3$ and $v 1+2 v 2+v 3$, the $v 1+2 v 2+v 3$ band has the greatest absorption cross-section.

\section{System Architecture}

The $\mathrm{CO}_{2}$ measurement system will use 126 path-integrated laser absorption measurements, arranged into 6 regularlyspaced projections of 21 beams at $75 \mathrm{~mm}$ pitch. Alternating the propagation direction between adjacent beams provides additional, but probably unnecessary, protection against crosstalk. The measurement region, defined by the intersection of these projections, extends beyond the hot exhaust zone sufficiently that the peripheral sections of each beam are inactive with regard to the spectroscopy of the chosen absorption line, rendering the system insensitive to ambient $\mathrm{CO}_{2}$.

The system is functionally equivalent to 126 single-path TDLAS systems but the cost of 126 diode lasers and their associated temperature and current controllers is obviously prohibitive. Instead a Master Oscillator Power Amplifier (MOPA) approach has been implemented, in which a single DFB diode laser seed is amplified by a Thulium-Doped Fiber Amplifier (TDFA). The TDFA output retains the amplitude and wavelength modulation properties of the seed but at optical power levels up to $2.5 \mathrm{~W}$. Passive splitters are then used to output the required 126 optical channels over single mode (SM) fiber at power levels consistent with shot noise limited detection performance. This Tunable Fiber

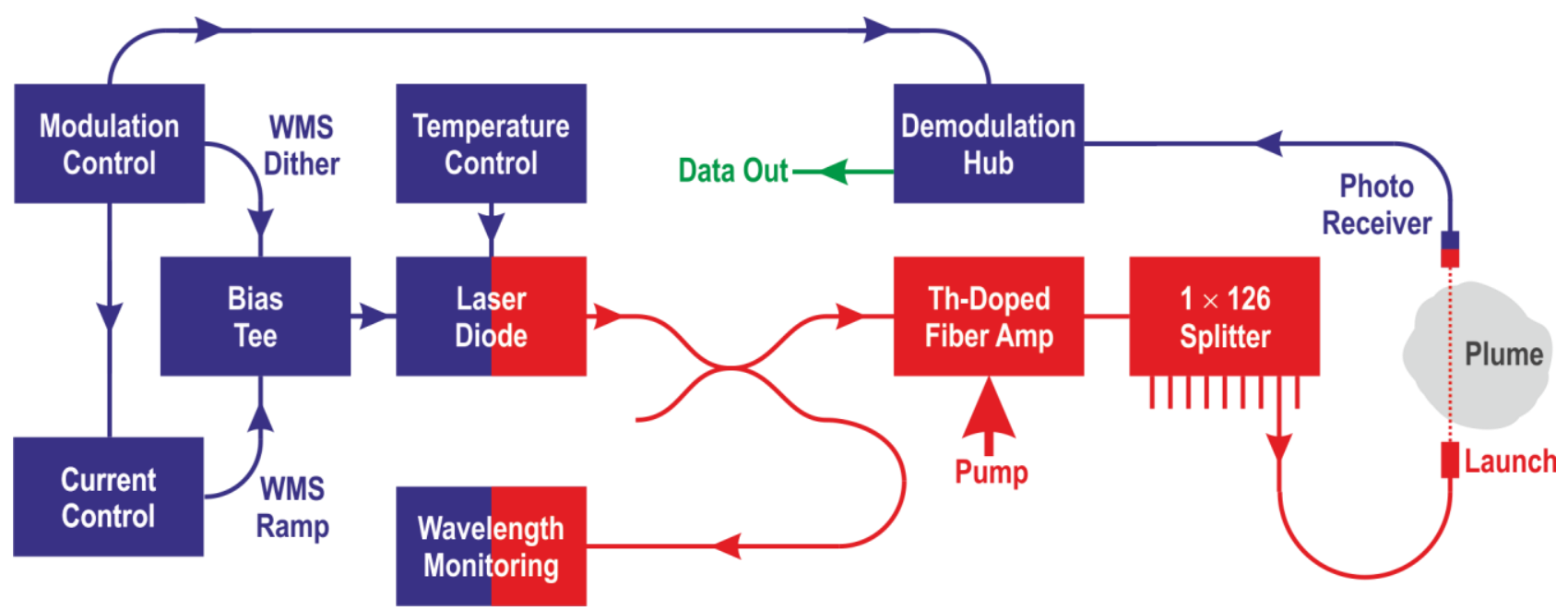

Figure 4 - TFLAS architecture. Colors denote electronic (blue), optical (red), and data (green) connections.

A soon-to-be-published experimental characterization of the selected absorption line was then undertaken, considering temperature, pressure and broadening effects. Using the resulting parameters, laboratory TDLAS tests, using a $5.5 \mathrm{~cm}$ pathlength of $100 \% \mathrm{CO}_{2}$ at $300 \mathrm{mbar}$ and $600^{\circ} \mathrm{C}$ to provide a realistic path-integrated transmittance, could be fitted with rms residual errors of less than $0.1 \%$, in comparison to a $3.5 \%$ transmittance change.
Laser Absorption Spectroscopy (TFLAS) arrangement, illustrated in Figure 4, constitutes a significant technological advance; the need to share a small number of diode laser sources across many beam paths has been performance limiting in previous optical absorption tomography systems, e.g. that used in [18], necessitating considerable effort in receiver amplifier design [19]. The system will implement a WMS detection scheme, acquiring one hundred spectral scans per second per beam path, using an adjustable dither frequency in the range 50 to $500 \mathrm{kHz}$. 


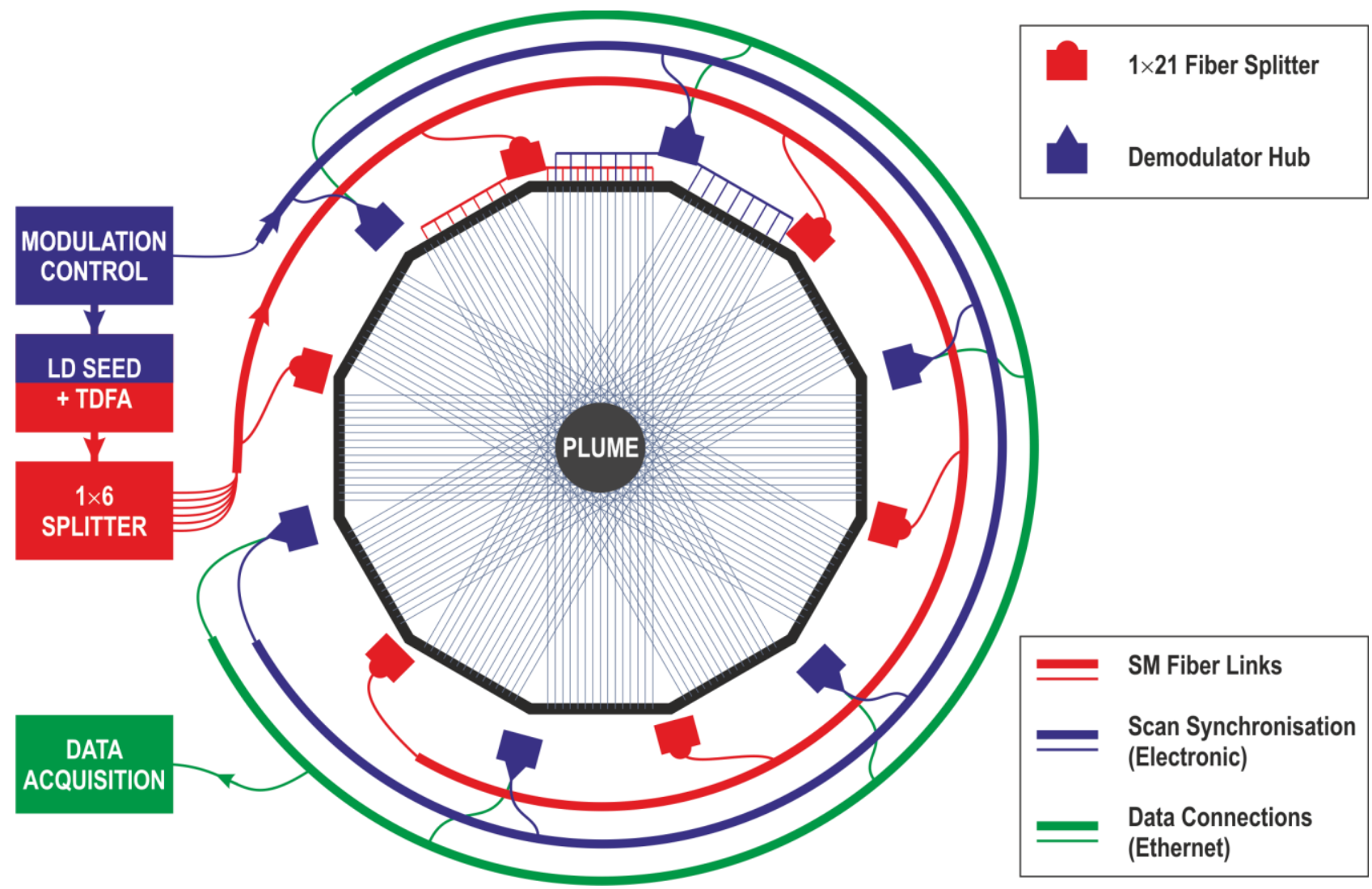

Figure 5 - Concept drawing of the dodecagonal mounting frame showing fiber-based light delivery (red), electronic signal connections (blue) and Ethernet data transfer (green). For clarity, the interleaved projection level connections are shown for only one fiber splitter and an adjacent demodulator hub.

The outputs from the 126 individual photoreceivers, each consisting of an extended InGaAs photodiode, transimpedance amplifier and line driver, will be grouped together in 21-channel blocks to enable efficient implementation of the sampling and digital demodulation electronics. Each of these six hubs receives timing signals to enable synchronization with the scanning cycle of the seed laser. Two Ethernet interfaces per hub are used for data output to remote PCs. Figure 5 shows the proposed system architecture.

\section{Hub Design}

Each hub serves 21 photoreceivers, drawn from the two projections adjacent to the vertex where it is mounted. Each photoreceiver output will be sampled at 40 MSPS, with up to 14 bit resolution, leading to a total data rate exceeding $10 \mathrm{~Gb} \mathrm{~s}^{-1}$ per hub, making local digital demodulation and subsequent decimation essential. This demodulation process is the principal function of the field programmable gate arrays (FPGAs) within each hub. I/Q demodulation will be performed at $1 f$ and $2 f$, where $f$ is the WMS dither frequency, generating four 32-bit values for each point of the wavelength scan. Each $10 \mathrm{~ms}$ scan comprises 500 such points, resulting in a post-demodulation hub aggregate data rate of around $128 \mathrm{Mb} \mathrm{s}^{-1}$. The use of two ports per hub avoids reliance on gigabit Ethernet, even once protocol overheads are accounted for. The use of digital modulation control and demodulation permits frequency agility, potentially allowing the WMS measurement to be made in a favorable spectral region for the prevailing engine operating conditions. The inherent parallelism of FPGAs has led to their use in numerous multichannel digital demodulation systems, e.g. [20]. 32 measurement channels are implemented per hub, providing 11 additional channels for either simultaneous operation of the UHC system, or future expansion of the $\mathrm{CO}_{2}$ system.

The optical power available from the TDFA is sufficient to provide SNR in excess of $80 \mathrm{~dB}$ at each photoreceiver output. Realizing the full potential of these signals makes the hub a challenging mixed signal design, requiring coexistence of these high SNR analogue signals and a relatively low jitter (<10 ps) sampling clock with no less than 40 high speed digital serial lines, each operating at 560 Mbps.

\section{Single Channel Engine Tests}

Single channel $2 f / 1 f$ TDLAS measurements, carried out in an aero-engine exhaust plume, have confirmed the viability of the proposed measurement approach (Figure 6). 


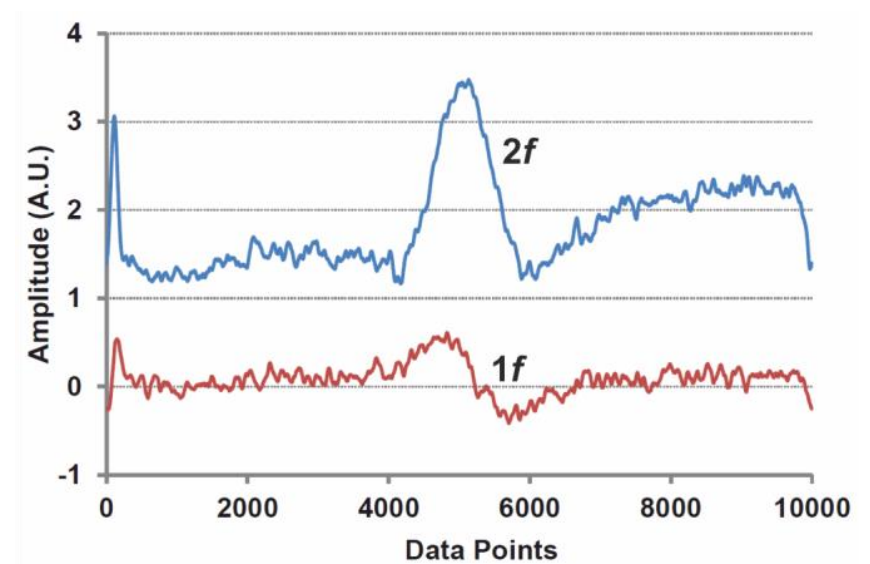

Figure 6 - Second harmonic signal (blue), X-channel, with the residual Y-channel signal (red). At $50 \mathrm{kHz}$ modulation frequency the signal requires significant averaging to remove excess noise (64 averages).

\section{Demonstration of TFLAS}

In what we believe is the first demonstration of its kind, a TDFA amplified seed has been used to make direct and $2 f$ WMS measurements of $\mathrm{CO}_{2}$ under laboratory conditions (Figure 7). A full characterization of the TDFA's response under a range of seed modulation conditions is now underway.
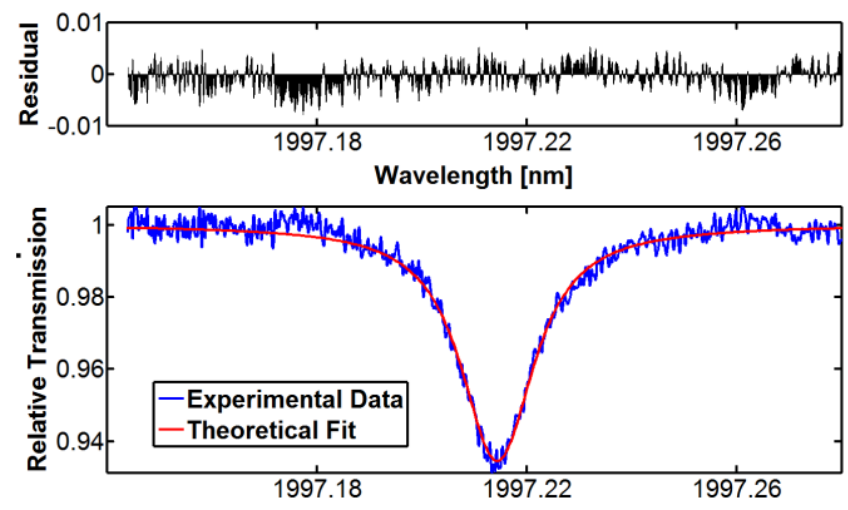

Figure 7 - Direct measurement of $\mathrm{CO}_{2}$ line using a TDFA operating just above threshold. Phantom is $100 \%$ $\mathrm{CO}_{2}$ at 300 mbar and $373 \mathrm{~K}$.

\section{UHC MEASUREMENT}

The mid-IR region offers tremendous opportunities for the observation of many chemical species. The higher linestrengths typical of this spectral region are undeniably advantageous at the low concentrations relevant to emissions monitoring but the region is technologically less accessible than the near-IR. The proposed UHC measurement system is intended to target several unburnt or partially decomposed hydrocarbon species, using wavelengths in the vicinity of $3.3 \mu \mathrm{m}$.

The proposed architecture is similar to that of the $\mathrm{CO}_{2}$ system, but using 6 projections of 8 beams each. The mounting frame and demodulation hubs are also shared but dedicated light generation, distribution and detection systems are required.

\section{Spectroscopy}

The transient spectroscopy techniques required for some of the target species of interest lie outside of the FLITES consortium's expertise. The spectroscopic parameters of these species will be characterized in the group of Professor Ron Hanson at Stanford University.

\section{Glass and Fiber Development}

The lack of suitable mid-IR glasses for use in fiber amplification or distribution is indicative of the less developed nature of mid-IR technologies and is a major barrier to the use of high channel count mid-IR systems. To date, a variety of IR materials have been drawn into optical fiber. These include the heavy metal fluoride glasses (HMFG), chalcogenide and polycrystalline fibers as well as hollow rectangular waveguides [21]. While none of these fibers had physical properties even approaching those of conventional silica fibers, they are, nevertheless, useful in lengths less than 2 to $3 \mathrm{~m}$ for a variety of IR sensor and power delivery applications.

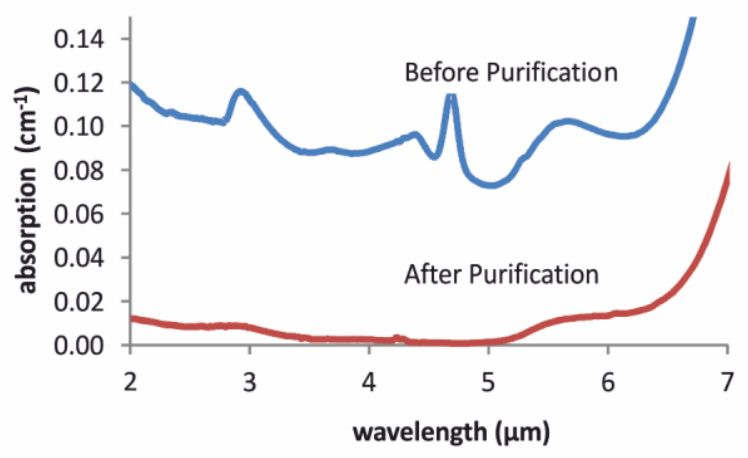

Figure 8 - Purification processes introduced during chalcogenide glass melting have led to significant improvements in glass transmission, with absorption peaks significantly reduced and an overall reduction in background scattering (this work).

For the purposes of this project we have identified chalcogenide glasses as the most promising material, offering higher temperature stability than other mid-IR materials and the potential for longer, lower loss fibers providing suitable glasses can be obtained. Our work therefore includes development of high purity chalcogenide glasses, suitable for drawing into optical fiber, for applications in the 2 to $5 \mu \mathrm{m}$ region. We have focused on the chalcogenide glass family based on gallium lanthanum sulfide (Ga:La:S) which offers several advantages over other commercially available chalcogenides. Commercial chalcogenide glasses are typically based on toxic arsenic, selenium and/or tellurium and melt at temperatures below $500^{\circ} \mathrm{C}$. The Ga:La:S glasses however contain no toxic elements and have the highest temperature stability of any 
chalcogenide making them better suited for harsh environments [22]. To date, substantial progress has been made through the implementation of a gas delivery system which provides a dry, high purity atmosphere under which glasses are melted. Background scattering loss has been lowered by a factor of 100 and, crucially for UHC applications, moisture impurities have also been substantially reduced, improving transparency around $3 \mu \mathrm{m}$. In addition, the infrared absorption edge has been pushed beyond $5 \mu \mathrm{m}$ by reducing oxide impurities, opening up much of the mid-IR window, including that needed for $\mathrm{NO}_{\mathrm{X}}$ measurement (Figure 8). Fiber drawing trials of these glasses are now underway.

\section{Light Sourcing and Distribution}

In recent years, mid-IR sources have seen considerable development with improvements in output power and increasing accessibility of longer wavelengths. Quantum cascade lasers have become preeminent, despite being comparatively inefficient and expensive. Direct bandgap III$\mathrm{V}$ semiconductor diode lasers can operate in the range of 2$3 \mu \mathrm{m}$ at room temperature but are limited at longer wavelengths, due to Auger recombination. Ceramic lasers based on for example, $\mathrm{Cr}^{2+}: \mathrm{ZnSe}$ are commercially available but thermal lensing limits the output power. In addition to providing an excellent solution for near-IR sensing of $\mathrm{CO}_{2}$, fiber lasers are strong candidates for mid-IR applications, offering significant advantages over alternative sources [23]. lasing has been proven at 2.7 and $3.6 \mu \mathrm{m}$ and research continues in parallel with our activities on up-doped fiber for mid-IR transmission (Figure 9).

Of greater concern are the difficulties apparent in distributing the light to the required beam launch locations. The scale of the mounting frame is such that fiber lengths of at least $10 \mathrm{~m}$ must be expected. Even our lowest bulk glass losses to date correspond to less than $1 \%$ transmittance over such a fiber at $3.3 \mu \mathrm{m}$, suggesting that fiber-based distribution may be unrealistic at this wavelength with present technologies, despite the substantial progress that has been made in suppressing the $3 \mu \mathrm{m}$ water peak.

\section{Soot Measurement}

LII has been applied to soot measurement for more than three decades [25]. In-situ LII studies of aero-engine exhaust plumes have been undertaken, including some incorporating scanning arrangements to provide rudimentary spatial insight, e.g. [26, 27]. These scanned studies relied on detection of the backward scattered component of the incandescence so their outputs show the angular variation of path-integrated incandescent emission, rather than its 2-D distribution. FLITES aims to combine fiber laser technology with tomographic techniques, first demonstrated in fluorescence tomography [28, 29], to achieve true crosssectional imaging of the soot distribution in the plume. The proposed method involves the use of a scanned excitation
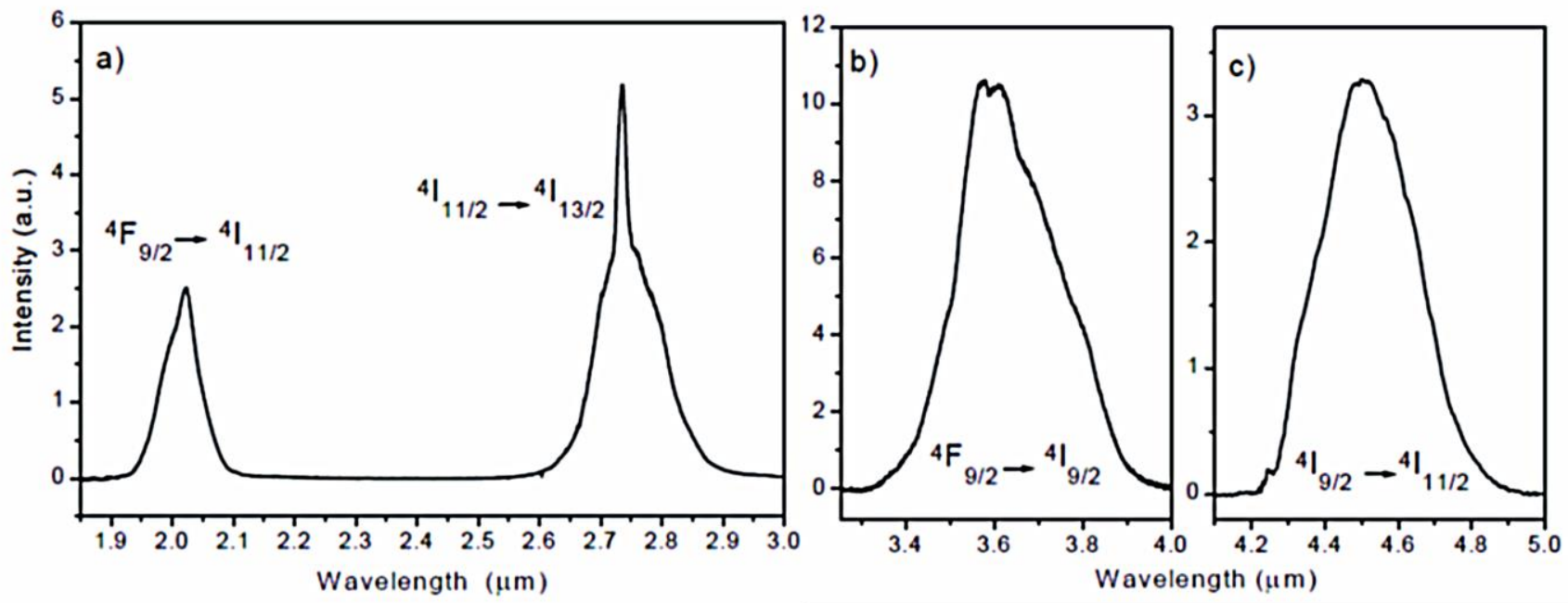

Figure 9 - Fluorescence spectra of Er3+ doped chalcogenide glass showing (a) $2.0 \mu \mathrm{m}$ and $2.75 \mu \mathrm{m}$ emission and (b) $3.6 \mu \mathrm{m}$ emission when pumped with $60 \mathrm{~mW}$ at $660 \mathrm{~nm}$ and (c) $4.5 \mu \mathrm{m}$ emission when pumped with $570 \mathrm{~mW}$ at $810 \mathrm{~nm}[24]$.

Ga:La:S glasses offer an ideal host for rare earth doping. Unlike other chalcogenides, the lanthanum in these glasses can be easily substituted by any of the rare earth ions including erbium, thulium, holmium and dysprosium, all of which offer mid-IR transmission [24]. Although at an early stage, this project is currently evaluating erbium in particular, as a possible fiber laser dopant. The potential for laser with 'near-orthogonal' detection using one or more gated intensified CCD cameras (Figure 10). The use of cameras avoids the need to align many individual point detectors with the scan plane of the excitation source. In this 'autoprojection' approach to tomographic imaging the spatial origin of an LII signal is determined by considering the intersection of the laser path responsible for its 
generation with the relevant pixel's field-of-view.

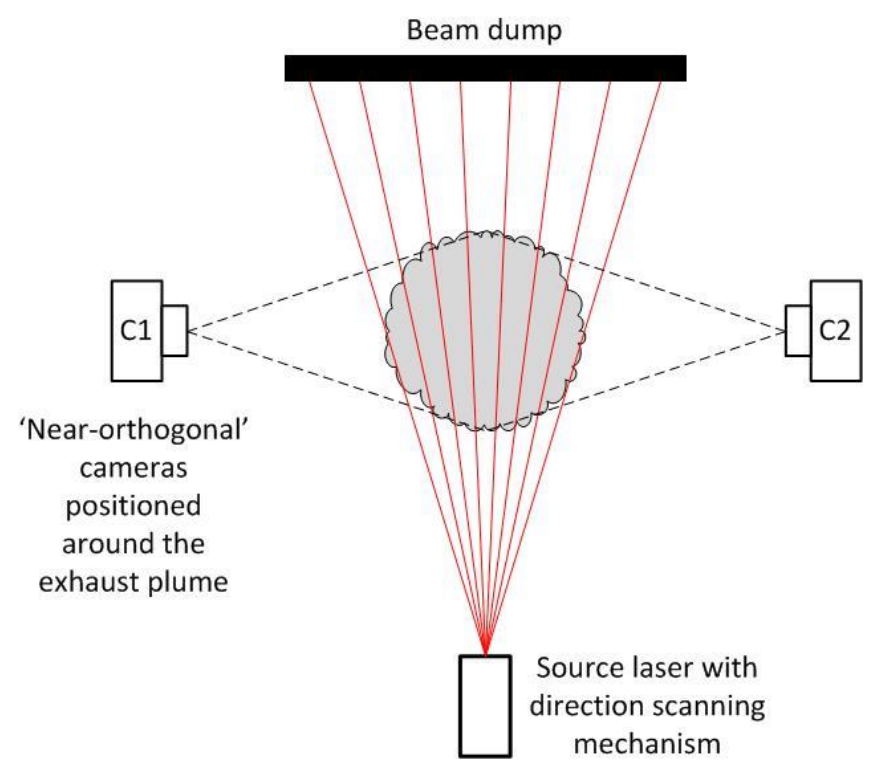

Figure 10 - Proposed LII imaging geometry

The Q-switched Nd:YAG lasers typically used to excite soot particles in LII studies provide short $(\sim 10 \mathrm{~ns})$ pulse durations at modest $(\sim 10 \mathrm{~Hz})$ repetition rates. We have investigated the use of fiber lasers, which are able to offer much higher repetition rates and are therefore better suited to imaging applications. We have been able to demonstrate both long and short pulse LII using fiber laser sources.

Long Pulse-Although the physics of long pulse LII is less well developed, the technique promises a significant sensitivity advantage over the conventional short pulse variant [30]. We have observed LII in both a jet exhaust plume and ambient air using a gated intensified CCD camera (Andor DH734) in conjunction with a fiber laser of $192 \mathrm{~ns}$ pulse width. These demonstrations used average powers of either $2.2 \mathrm{~W}$ or $11 \mathrm{~W}$, well below the laser's $30 \mathrm{~W}$ capability, and a repetition rate of $30 \mathrm{kHz}$. The camera's field-of-view was arranged orthogonal to the excitation beam, in anticipation of the proposed tomographic arrangement.

Short Pulse - The physics of LII generated from short laser pulses, of the order of $10 \mathrm{~ns}$, is well understood from the literature. Fiber lasers have several potential advantages for short pulse LII studies, including high power and repetition rate, as well as the potential for temporal pulse shaping. We have recently demonstrated short pulse LII, at a $10 \mathrm{kHz}$ pulse repetition rate, using a fiber laser of $17 \mathrm{~ns}$ pulse width. To date, this system has only been deployed in laboratory studies.

\section{SUMMARY}

Measurement technology has an important role to play in supporting the transition to more sustainable civil aviation. To this end, the FLITES project is attempting to develop tools for imaging the cross-sectional distributions of both major constituent $\left(\mathrm{CO}_{2}\right)$ and minor pollutant (UHC, soot) species within jet exhaust plumes. The program has already demonstrated the viability of key elements of these three measurement systems, including TDFA-based TFLAS for $\mathrm{CO}_{2}$ quantification and both short and long pulse fiber LII.

Two of the three systems $\left(\mathrm{CO}_{2}\right.$, soot $)$ are now moving into their implementation phases. At the time of writing, construction of the dodecagonal mounting frame is imminent and contracts for the manufacture of the associated passive fiber optic splitting/distribution system and electrical wiring harnesses are being negotiated.

The spectroscopic aspects of the $\mathrm{CO}_{2}$ measurement system are established and TFLAS has been demonstrated. The system's electronic design is in the layout phase and most elements of its firmware are at an advanced stage of development. The finished system will implement the core functionality of 126 conventional TDLAS systems, including the high-bandwidth lock-in amplifiers needed for $2 f / 1 f$ WMS. The synchronization and data communication mechanisms required by the distributed nature of the system, and its high aggregate output data rate (around $\left.1 \mathrm{~Gb} \mathrm{~s}^{-1}\right)$, are yet to be tested.

It appears unlikely that we will deliver a full UHC imaging system within the present project, as sufficiently low-loss and low-cost fiber remains elusive, although very significant progress towards realizable mid-IR systems has been made. It is nonetheless expected that the program will deliver proof-of-principle, in the form of i) relevant spectroscopy, ii) a single UHC measurement channel and iii) an evaluation of the feasibility of fiber delivery, at both $3.3 \mu \mathrm{m}$ and elsewhere in the mid-IR region.

Progress towards LII system implementation has focused on source development, supported by fixed path tests on both laboratory phantoms and jet plumes. The demonstration of long pulse LII in a jet plume is a significant first. Extension of these single path measurements to a full LII imaging capability requires comparatively little additional hardware development, primarily the introduction of suitable optical scanning and timing synchronization arrangements, but some further work is needed in regard of system modelling and calibration. Overall, however, there appears to be no fundamental barrier to successful implementation of LIIbased soot imaging.

In conclusion, the project is on track to deliver crosssectional imaging of both $\mathrm{CO}_{2}$ and soot in jet exhaust plumes, as well as substantial advancement of mid-IR technologies that will eventually permit tomographic imaging of pollutant species such as $\mathrm{UHCs}_{\mathrm{N}}$ or $\mathrm{NO}_{\mathrm{X}}$. 


\section{ACKNOWLEDGEMENTS}

The authors wish to acknowledge Royal Dutch Shell plc, and in particular Joanna Bauldreay, for their support in respect of the conventional and alternative aviation fuel aspects of this work. The authors are also grateful to those staff of the Instituto Nacional de Tecnica Aeroespacial (INTA) who have contributed to the design of the mounting frame and to the industrial partners within the FLITES consortium for their various contributions to the project. This work is funded by the UK Engineering \& Physical Sciences Research Council.

\section{REFERENCES}

[1] P. Walker, "Third runway plan for Heathrow scrapped by BAA," in The Guardian, ed. www.theguardian.com, 2010.

[2] "The Environmental Effects of Civil Aircraft in Flight," ed: Royal Commission on Environmental Protection, 2002.

[3] "SAE ARP 1179 Aircraft Gas Turbine Engine Exhaust Smoke Measurement, Aerospace Recommended Practice," ed: SAE International, 1997.

[4] K. Schäfer, J. Heland, D. H. Lister, C. W. Wilson, R. J. Howes, R. S. Falk, et al., "Nonintrusive optical measurements of aircraft engine exhaust emissions and comparison with standard intrusive techniques," Applied Optics, vol. 39, pp. 441-455, 2000.

[5] "Regulation (EC) No 715/2007 of the European Parliament and of the Council of 20 June 2007 on type approval of motor vehicles with respect to emissions from light passenger and commercial vehicles (Euro 5 and Euro 6) and on access to vehicle repair and maintenance information," ed. Eur-lex.europa.eu, 2007.

[6] "How to comply with your environmental permit. Additional guidance for: The Incineration of Waste (EPR 5.01)," UK Environment Agency, 2009.

[7] S. Bainbridge, "Emissions Verification in the Aviation Sector " Lucideon CICS Ltd., April 2014.

[8] S. J. Carey, H. McCann, F. P. Hindle, K. B. Ozanyan, D. E. Winterbone, and E. Clough, "Chemical species tomography by near infra-red absorption," Chemical Engineering Journal, vol. 77, pp. 111-118, Apr 152000.

[9] F. P. Hindle, S. J. Carey, K. Ozanyan, D. E. Winterbone, E. Clough, and H. McCann, "Measurement of gaseous hydrocarbon distribution by a near-infrared absorption tomography system," Journal of Electronic Imaging, vol. 10, pp. 593-600, Jul 2001.
[10] D. McCormick, M. G. Twynstra, K. J. Daun, and H. McCann, "Optimising laser absorption tomography beam arrays for imaging chemical species in gas turbine engine exhaust plumes," in WCIPT7: 7th World Congress on Industrial Process Tomography, Krakow, Poland, 2013, pp. 505-514.

[11] C. B. Hogge and W. L. Visinsky, "Laser Beam Probing of Jet Exhaust Turbulence," Applied Optics, vol. 10, pp. 889-892, 1971.

[12] M. Henriksson, L. Sjöqvist, and O. Gustafsson, "Experimental study of mid-IR laser beam wander close to a jet engine exhaust. Proc. SPIE 6397, Technologies for Optical Countermeasures III, 639709 (October 05, 2006); doi:10.1117/12.689638.," 2006.

[13] W. M. Isterling, B. B. Dally, Z. T. Alwahabi, M. Dubovinsky, and D. Wright, "Beam displacement as a function of temperature and turbulence length scale at two different laser radiation wavelengths," Applied Optics, vol. 51, pp. 55-63, 2012.

[14] V. S. Sirazetdinov, "Experimental study and numerical simulation of laser beams propagation through the turbulent aerojet," Applied Optics, vol. 47, pp. 975-985, 2008/03/01 2008.

[15] P. Wright, E. Fisher, and H. McCann, "Optical Design Considerations for Chemical Species Tomography in a Jet Exhaust Plume," in Imaging and Applied Optics 2014, Seattle, Washington, 2014, p. JTu4A.2.

[16] "ICAO Assembly Resolution A37-19," International Civil Aviation Organization, 2010.

[17] L. S. Rothman, I. E. Gordon, A. Barbe, D. C. Benner, P. F. Bernath, M. Birk, et al., "The HITRAN 2008 molecular spectroscopic database," Journal of Quantitative Spectroscopy and Radiative Transfer, vol. 110, pp. 533572, 2009.

[18] P. Wright, N. Terzija, J. L. Davidson, S. Garcia-Castillo, C. Garcia-Stewart, S. Pegrum, et al., "High-speed chemical species tomography in a multi-cylinder automotive engine," Chemical Engineering Journal, vol. 158, pp. 2-10, Mar 152010.

[19] P. Wright, K. B. Ozanyan, S. J. Carey, and H. McCann, "Design of high-performance photodiode receivers for optical tomography," IEEE Sensors Journal, vol. 5, pp. 281-288, Apr 2005.

[20] S. J. Lascos and D. T. Cassidy, "Multichannel digital phase sensitive detection using a field programmable gate array development platform," Review of Scientific Instruments, vol. 79(7), 2008. 
[21] Harrington, J. A., "Selected Papers on Infrared Fiber Optics," Milestone Series, Volume MS-9," SPIE Press, Bellingham, WA, SPIE Press, Bellingham, WA, 1990.

[22] D. W. Hewak, "The promise of chalcogenides," Nat Photon, vol. 5, pp. 474-474, 2011.

[23] S. D. Jackson, "Towards high-power mid-infrared emission from a fibre laser," Nat Photon, vol. 6, pp. 423431, 2012.

[24] T. Schweizer, "Rare-earth-doped gallium lanthanum sulphide glasses for mid-infrared fibre lasers," $\mathrm{PhD}$, University of Southampton, 2000.

[25] L. A. Melton, "Soot diagnostics based on laser heating," Applied Optics, vol. 23, pp. 2201-2208, 1984.

[26] J. D. Black and M. P. Johnson, "In-situ laser-induced incandescence of soot in an aero-engine exhaust: Comparison with certification style measurements," Aerospace Science and Technology, vol. 14, pp. 329-337, 2010.

[27] J. D. Black, J. Delhay, P. Desgroux, and M. P. Johnson, "In-Situ Laser-Induced Incandescence of Soot in Large Civil Aeroengine Exhausts," in 26th AIAA Aerodynamic Measurement Technology and Ground Testing Conference, ed: American Institute of Aeronautics and Astronautics, 2008.

[28] K. B. Ozanyan and H. McCann, "Efficiency and data correction for OFAPT sensors with fiber receivers," IEEE Sensors Journal, vol. 5, pp. 195-202, Apr 2005.

[29] F. Hindle, H. McCann, and K. B. Ozanyan, "First demonstration of optical fluorescence auto-projection tomography," Chemical Engineering Journal, vol. 77, pp. 127-135, Apr 152000.

[30] D. McCormick, K. B. Ozanyan, J. D. Black, and F. Yutong, "In-situ soot particle sensing in an aero-engine exhaust plume," in IEEE Sensors 2013, pp. 1254-1257.

\section{BIOGRAPHY}

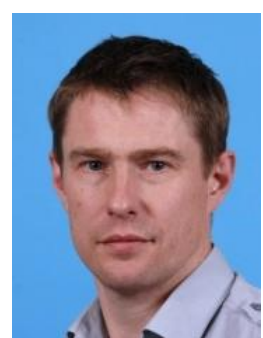

Paul Wright received the B.Sc. degree in pure and applied physics from the University of Nottingham, U.K., and the Ph.D. degree in physics from the University of Exeter, U.K., in 1993 and 1997, respectively. He was a Lecturer in the Division of Measurement and Control, University of Teesside, before spending five years with Land Infrared as a Project Engineer working primarily on industrial applications of optical radiometry. He joined the University of Manchester, Manchester, U.K., in 2002, where he is currently a Lecturer. He has designed numerous optical and electronic instruments and coauthored publications on near-IR thermometry, optical tomography and medical instrumentation. His work has been recognized with the Maurice Beck Prize for best paper at the 5th WCIPT (Bergen, 2007) and best poster at Photon 06 (Manchester, 2006).

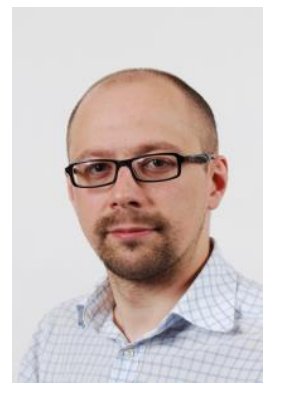

David McCormick (M'13) received the B.Eng. degree in electronic systems engineering and the Eng.D. degree in electrical and electronic engineering from the University of Manchester, UK, in 2006 and 2011 respectively. He also received the Pg.Dip degree in management sciences from Manchester Business School in 2008. Following 10-years working in industry for several large engineering organizations and UK-based SMEs, between 2012 and 2014 he was a postdoctoral researcher working on the FLITES project. Since 2014, he has been a Lecturer with the School of Electrical and Electronic Engineering at the University of Manchester, UK. His research interests include aerospace combustion diagnostics and optical and electrical tomography. Dr. McCormick is a Chartered Engineer with the Institution of Engineering and Technology, is the current holder the Worshipful Company of Scientific Instrument Makers Beloe Fellowship and is a Whitworth Senior Scholar. His awards include the Whitworth Visionary Award 2012 and a Whitworth Senior Scholarship Award (both from the Institution of Mechanical Engineers).

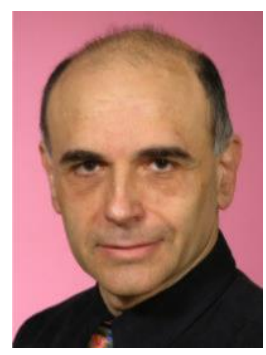

Krikor B. Ozanyan (M'1995, SM'2003) received his $M S c$ degree in engineering physics (semiconductors) and $\mathrm{PhD}$ degree in solid-state physics, in 1980 and 1989 respectively, from the University of Sofia, Bulgaria. He has held previous academic and research posts in the University of Sofia, The Norwegian Institute of Technology (Trondheim, Norway), the University of Hull (UK), and the University of Sheffield $(U K)$, working on projects ranging from Brewster-angle 
mid-IR spectroscopic ellipsometry and electron confinement in quantum wells and barriers, to the demonstration of the lasing at 333nm from strained $M Q W$ $\mathrm{ZnCdS/ZnS} \mathrm{structures} \mathrm{and} \mathrm{in-situ} \mathrm{real-time} \mathrm{optical}$ monitoring of growth of III-V semiconductors in MBE and MOCVD reactors. His current interests are in the area of photonic sensors and indirect imaging (tomography) by optical modalities, signal processing for optical experiments, and spectroscopy with ultrafast laser sources. He is currently Head of Sensors, Imaging and Signal Processing at the University of Manchester and Visiting Professor at the University of Bergen, Norway. Professor Ozanyan is Fellow of the Institute of Engineering and Technology, (UK, formerly IEE) and Fellow of the Institute of Physics (UK). He was Distinguished Lecturer of the IEEE Sensors Council in 2009-2010, Guest Editor of the IEEE Sensors Journal Special Issues "Sensors for Industrial Process Tomography" in 2005 and "THz Sensing: Materials, Devices and Systems" in 2012. He is currently Editor-inChief of the IEEE Sensors Journal.

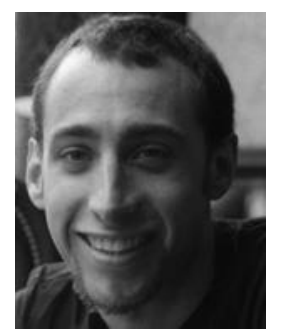

Edward Fisher (M'08) works as a post-doctoral research assistant in the Agile Tomography group within the institute of digital communications (IDCOM). His specialisation and research centre on imaging systems from single-photon sensor development, through to data acquisition and signal processing. He has a particular interest in modelling and optimisation for system design, along with specifications engineering and the implementation of reconfigurable, agile distributed data acquisition and computation systems. Recently centring on mixed-signal design, he has concentrated on including design for test, design for manufacture and design for mechanical considerations, into system development.

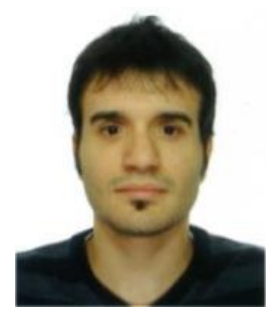

Andrea Chighine received the B.Sc. degree in electrical engineering and M.Sc. degree in telecommunication engineering from the University of Rome "Roma Tre" Roma, Italy in 2009 and 2012, respectively. Between 2011 and 2012, he was an intern master student with the Photonic Integration group at the Eindhoven University of Technology (TU/e), Eindhoven, The Netherlands. Since January 2013, he has been with the Agile Tomography research group at the University of Edinburgh, Edinburgh, U.K. and working toward the Ph.D. degree, where his work focuses on the digital electronic design within the FLITES project.

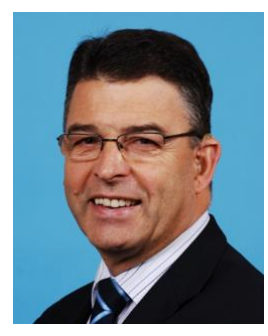

Hugh McCann received the B.Sc. and Ph.D. degrees from the University of Glasgow, Glasgow, U.K., in 1976 and 1980, respectively. He was appointed Professor of Tomographic Imaging and Head of the School of Engineering at The University of Edinburgh, Edinburgh, Scotland, U.K. in 2013. From 1996 - 2013, he was Professor of Industrial Tomography at the University of Manchester, Manchester, U.K., following ten years in $R \& D$ at the Royal Dutch/Shell Group. He worked in highenergy particle physics for ten years at Glasgow, Manchester, CERN (Geneva, Switzerland) and DESY (Hamburg, Germany). He has extended industrial tomography to provide specific chemical contrast using high-speed all-opto-electronic techniques, and has developed electrical impedance tomography for medical applications, collaborating intensively with users in both academia and industry. He was Head of the School of Electrical and Electronic Engineering at Manchester (1999-2002), then chaired the U.K. Professors and Heads of Electrical Engineering (2003-2005) and the Virtual Centre for Industrial Process Tomography (20052009). Prof. McCann was elected a Fellow of the Royal Academy of Engineering in 2009.

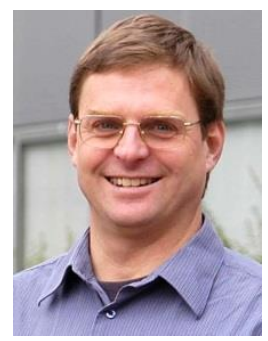

Daniel W Hewak was born in Canada where he received the B.SC. Degree in Physics from McMaster University, Hamilton, Ontario in 1982 and Ph.D. from the University of Waterloo, Waterloo, Ontario in 1988. In 1988 he joined the National Optics Institute in Ste. Foy, Quebec research scientist in particular working on industrial sponsored projects from IBM and Digital Equipment of Canada. In 1991 he left Canada to take a research position at the Optoelectronics Research Centre, University of Southampton in the United Kingdom. He now specializes in the production and application of chalcogenide glass and is recognized internationally as a leader in the production and use of chalcogenides and a pioneer in their fabrication and application. His research has led to IR fibers for sensing, medical and aerospace, fiber amplifiers for telecom, the first chalcogenide-glass lasers and also solar-cell, thermoelectric and memory devices. This diversity is a testament to the truly advanced functionality of chalcogenides. Over the past five years, Dan has contributed to the management of several large projects, notably the EPSRC Centre for Innovative Manufacturing in Photonics (CIMP). His research is well funded by industry, including Merck, Pirelli and DSTL and currently via Samsung's Global Research Outreach Program, and he works closely with Ilika Technologies Ltd on material development and characterization. He has authored over 270 papers and conference publications (h-index 34), holds 9 patents, with 3 licensed 
to industry.

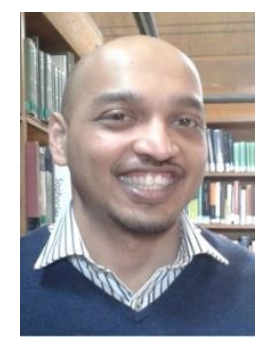

Khouler Khan was born in Trinidad where he received a B.Sc. Degree in Electrical and Computer Engineering from the University of the West Indies, Trinidad in 2003. He obtained and M.Sc. in Nano-electronics and mechanics from the University of Sheffield, United Kingdom in 2009 and is currently studying for his Ph.D. at the University of Southampton, United Kingdom. In 2004 he joined the Petroleum Company of Trinidad and Tobago in Trinidad as an Engineer and has worked on many large and small scale industrial projects in the Oil and Gas field. He now specializes in using his engineering and instrument talents to build innovative automated system solutions to improve the manufacturing of high purity infrared transmitting materials. Over the past four years, Khouler has made significant progress on improving the quality and production methods of infrared materials at the Optoelectronics Research Centre under the EPSRC Centre for Innovative Manufacturing in Photonics.

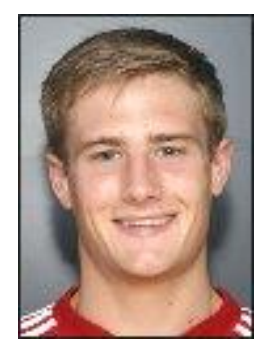

Paul Bastock graduated from the University of Glasgow with a Bachelor's in Mechanical Engineering with Aeronautics accompanied with the Highest Overall Engineering Degree Prize, 2011. In 2011, he joined the Optoelectronics Research Centre, University of Southampton working on novel fiber manufacture and funded through the EPSRC Centre for Innovative Manufacturing in Photonics. Research has focused in particular on specialty fiber manufacture and low loss mid-IR transmitting fiber for gas sensing and non-linear active fiber devices. Paul has worked as part of numerous industrial partnered projects and international collaborative research projects, most recently with Nanyang Technical University, Singapore. His research has been presented at five conferences so far throughout his PhD in the Optoelectronics Research Centre.

Johan Nilsson is a Professor at the Optoelectronics Research Centre (ORC), University of Southampton, England. In 1994, he received a doctorate in Engineering Science from the Royal Institute of Technology, Stockholm, Sweden, for research on optical amplification. Since then, he has worked on optical amplifiers and amplification in lightwave systems, optical communications, and guided-wave lasers, first at Samsung Electronics and later at ORC. His research has covered system, fabrication, and materials aspects of guided-wave lasers and amplifiers, and in particular device aspects of high power fiber lasers and erbiumdoped fiber amplifiers. He is leading ORC's high-power fiber laser group which, in recent years, demonstrated a number of world-firsts and world-records in the field, including the first single-mode $k W$ fiber laser and the first cladding-pumped fiber Raman laser. He has published nearly 400 scientific articles and has taught several short courses on fiber lasers and amplifiers. He is a fellow of the OSA, a life member of the SPIE, a consultant to, and co-founder of, SPI Lasers, and a member of the advisory board of the Journal of the Optical Society of Korea. In addition, he was guest editor of two issues on high-power fiber lasers in IEEE Journal of Selected Topics in Quantum Electronics in 2009. He has also been active in the organization of several conferences and meetings, and is a former chair of the Laser Science and Engineering technical group in OSA's Science and Engineering Council. At present he is a member of the technical program committee of the Europhoton and the Optical Instrument and Technology conference.

Michael Lengden received the M.Phys. degree in physics and the Ph.D. degree from the University of Manchester, Manchester, U.K., in 2001 and 2006, respectively, where he was engaged in stepwise excitation of atomic and molecular metastable states. He was an Applications Engineer at the Laboratory Impex Systems, Ltd., prior to becoming a Research Fellow in the Centre for Microsystems and Photonics at the University of Strathclyde between 2007 and 2010. He is currently a Lecturer in the EEE Department, University of Strathclyde, Glasgow, U.K., where he is engaged in hightemperature spectroscopy measurements using tunable diode laser spectroscopy. His current research interests include new techniques in tunable diode laser spectroscopy for concentration and pressure measurements in harsh environments, such as solid oxide fuel cells and aeroengines.

Walter Johnstone received the B.Sc. (Hons.) degree in chemical and material sciences and the Ph.D. degree in laser physics from the University of Strathclyde, Glasgow, U.K., in 1977 and 1982, respectively. From 1980 to 1986, he held Project Engineering and Senior Technical Management posts at Pilkington Optronics, Ltd. (now Thales) and Logitech, Ltd. In 1986, he joined the University of Strathclyde where he is currently Vice Dean of the Faculty of Engineering and a Professor of Photonic Systems in the Department of Electronic and Electrical Engineering. Since 1994, he has been a Director of OptoSci, Ltd., Glasgow, where he has been instrumental in bringing a number of photonics products to market. His work has led to more than 200 technical publications and several patent applications. His current research interests include optical waveguide components, fiber lasers, distributed fiber sensing systems, and optical gas sensing.

Ian Armstrong was awarded the B.Eng. (first class hons) degree in electronic and electrical engineering from the University of Strathclyde, Glasgow, Scotland, U.K., in 2001. For his research in semiconductor optical amplifier integration, in collaboration with Kamelian Ltd, he received the Eng.D. degree from the University of 
Strathclyde, Glasgow, Scotland, U.K., in 2006. Following a period as a Research Fellow at the Centre for Microsystems and Photonics at the University of Strathclyde, working on TDLS-based gas detection systems in the Near- and Mid-IR spectral regions, he joined OptoSci Ltd.. 